\title{
Effect of natural convection and radiation inside of a hollow beam in a standard fire
}

\author{
Turo Välikangas ${ }^{1} \quad$ Sami Pajunen $^{2} \quad$ Jolanta Baczkiewicz $^{2} \quad$ Singh Shobhana $^{3} \quad$ Kim Sørensen $^{3}$ \\ ${ }^{1}$ Laboratory of Chemistry and Bioengineering, Technical University of Tampere, \\ Finland,turo.valikangas@tut.fi \\ ${ }^{2}$ Laboratory of Civil Engineering, Technical University of Tampere, Finland sami . pa junen@tut . fi \\ ${ }^{3}$ Energy Technology, Aalborg University, Denmark ssi@et .aau.dk
}

\begin{abstract}
In the design of steel structures, special attention must be paid on structural fire design in order to ensure a specified safe time period that the structure can withstand the fire without collapse. In the European design rules, the standard practise assumes uniform temperature for steel beam cross sections while the surrounding area is subjected to the so called standard fire. When the ambient temperature field is not uniform (e.g. at beam joint areas) neither will be the beam cross section temperature field. This paper studies the contribution of natural convection and cavity radiation to the temperature field of a hollow beam cross section in the case of non-uniform ambient temperature by using transient CFD-simulations. Hollow beam,natural convection, cavity radiation, structural fire design, OpenFoam
\end{abstract}

\section{Introduction and background}

The current European standards for the structural fire design are given in Eurocode 1, Part 1-2 and in Eurocode 3, Part 1-2. In these design codes, the most common assumption is that the temperature field around the beam cross-section is uniform during the standard ISO-834 fire. Following that assumption, the structural design can be done in a straightforward manner, but it may result in a non-economic and too conservative solution. Particularly, steel beams are almost invariably assembled so that the fire does not affect equally on all the sides of the beam. Typically such situation arises in beam joint areas and in beam connections to surrounding structures. The Eurocode 3 includes some recommendations for open I-sections and steel slabs subjected to fire on three sides. However, no unified rules are available for the hollow sections in nonuniform surrounding temperature, and only some studies are available in the literature. One of the studies investigated steel pipes subjected to partial fire acting longitudinally along the pipe (Wong, 2017). In this study a parametrically coded generic element method was proposed to solve the heat transfer problem, which was more effective and accurate than Eurocode 3 calculations, but the paper did not consider the non-uniform temperature in the crosssection. The non-uniform heating of tubular sections was experimentally tested in (Yang et al., 2013) numerically in (Heinisuo and Jokinen, 2014) but only for concrete-filled columns. All above mentioned tests came to the same conclusion that the number of sides subjected to fire, has a great influence on the heat distribution and on the fire resistance.

In general, there is a large number of experimental and numerical research results concerning tubular sections in the uniform fire conditions, see e.g. (Fung et al., 2015),(Shao et al., 2016),(He et al., 2013) for circular hollow sections, and e.g. (Yang et al., 2014),(Ozyurt et al., 2014) for square hollow sections. Nevertheless, the nonuniform fire condition is far less studied, and to the authors' best knowledge there exist no reliable experimental results for the square hollow sections. Numerical studies are also rather few, the analytic approach of (Mills, 1999) can be used for radiation calculations, but for the natural convection only simplified geometries and numerical boundary conditions are covered (Kays, 2012). The natural convection inside a cavity is affected by the ratio between the height and the width of the cavity as well as the inclination of the roof wall. Temperature differences and their influence on the Rayleigh number of the situation has a significant effect on the turbulence level in the flow. This is why a three dimensional computational fluid dynamics approach was chosen in this study. This way the difference between convective and radiation heat transfer can be simulated in the laminar, transition and in the turbulent regime.

In this paper, the effects of natural convection and surface-to-surface radiation inside the square hollow beam due to the non-uniform fire condition are studied and some recommendations about their importance are given. On the other hand, the paper presents modelling principles for taking these phenomena into account in the structural fire design.

\section{Model development}

\subsection{Governing equations and boundary condi- tions}

1. Continuity equation 


$$
\frac{\partial \rho}{\partial t}+\frac{\partial\left(\rho u_{i}\right)}{\partial x_{i}}=0
$$

2. Momentum equation

$$
\frac{\partial \rho u_{i}}{\partial t}+\frac{\partial\left(\rho u_{i} u_{j}\right)}{\partial x_{j}}=\frac{\partial}{\partial x_{i}}\left(\mu \frac{\partial u_{j}}{\partial x_{i}}\right)-\frac{\partial p}{\partial x_{j}}
$$

where $u$ is the velocity and $x$ the coordinate location and where $\mathrm{i}, \mathrm{j}$ and $\mathrm{k}$ are the summation identities according Newton's formula in all three Cartesian coordinate directions. The others $\mathrm{t}, \mu$ and $\mathrm{p}$ are the time, dynamic viscosity and pressure.

3. Energy equation for fluid region

$$
\frac{\partial \rho T}{\partial t}+\frac{\partial}{\partial x_{i}}\left(\rho u_{i} T\right)=\frac{\partial}{\partial x_{i}}\left(\frac{\lambda_{f}}{C_{p}^{f}} \frac{\partial u_{i}}{\partial x_{i}}\right)
$$

where $T$ is the temperature, $C_{p}$ is the specific heat and $\lambda_{f}$ is the thermal conductivity.

4. Energy equation for solid region

$$
-\frac{\lambda_{s}}{C_{p}^{s}} \frac{\partial^{2} u}{\partial x_{i}^{2}}=0
$$

To validate how much heat is transferred through the boundary patch that is connecting the air and solid domain, conduction and radiation heat fluxes are calculated separately on the air. The conduction heat flux is calculated according to the equation 5 .

$$
\lambda_{f} \nabla T=0
$$

In this study radiation is calculated with a view factor model that says that the sum of all view factors from a given surface, $S_{i}$, is unity as explained in equation 6

$$
\sum_{n=1}^{i} F_{S_{i} \rightarrow S_{j}}=1
$$

To save computational resources an agglomeration script is ran before the calculation of the view factors. This means that the surfaces that are in the same plane and next to each other are embedded together because their view factors can be assumed to be equal. Then matrix system in equation 7

$$
Q_{\text {rad }}=C^{-1} b
$$

where $\mathrm{C}$ calculates the ratio between reflection and absorption and $\mathrm{b}$ is the emissivity matrix as follows.

$$
b=A e_{b}-H_{o}
$$

Where $\mathrm{A}$ is the view factor matrix that takes into account the agglomeration calculation and $e_{b}$ is the emissivity of a black surface. $H_{O}$ combines all the external radiation heat fluxes.

\section{Computational model}

In this section the computational model, the meshing process, solvers used and the grid independence study are illustrated.

\subsection{Computational geometry}

The simulated domain is divided in to two different regions. The hollow solid beam and the air inside the cavity. The solver that was used to solve the time dependent temperature equation and the three dimensional laminar flow field inside the hollow beam is chtMultiRegionFoam. Second order accurate upwind schemes where used for the velocity and temperature field and only first order accurate upwind for all the others. The coupling between momentum and pressure equation in chtMultiRegionFoam is solved using PIMPLE algorithm which is a combination between PISO (Pressure Implicit with Splitting of Operator) and SIMPLE (Semi-Implicit Method for Pressure Linked Equations) algorithms. For schemes a second order accurate upwind schemes was used for velocity and temperature equation and a first order upwind scheme was used for all the others. In figure 1 the computational domain on the solid side is illustrated. The boundary patches are called insulated, the cold wall, the warm wall and the solid to fluid patch according to the color coding in the figure.

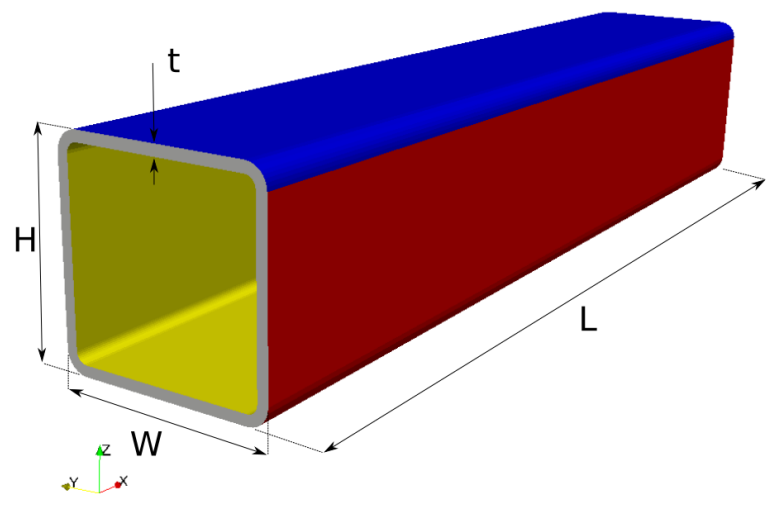

Figure 1. Illustration of the boundary patches on the solid region and the geometry variables

Table 1. Geometric dimensions of the hollow beam

\begin{tabular}{lrr}
\hline \multicolumn{1}{c}{ Parameter } & Symbol/unit & Value \\
\hline Length of the beam & $L / \mathrm{mm}$ & 500 \\
Height of the beam & $H / \mathrm{mm}$ & 100 \\
Width of the beam & $W / \mathrm{mm}$ & 100 \\
Thickness of the beam wall & $t / \mathrm{mm}$ & 5 \\
\hline
\end{tabular}


Because the patches of the meshes that are connecting the two regions together are not conformal, a interpolative mapping needs to be used for fields that are communicating . For this a boundary condition called mappedWall in OpenFOAM is used. (Foundation, 2017) To define how the information is mapped between the regions a sample mode can be chosen and for this study the nearestPatchFaceAMI(Arbitrary mesh interface) is used. This means that the information is exchanged between nearest faces and if the matching is not identical, weight functions are used. In figure 2 the computational domain for the air side is illustrated. The boundary patches are called insulated and fluid to solid patch as illustrated in the figure.

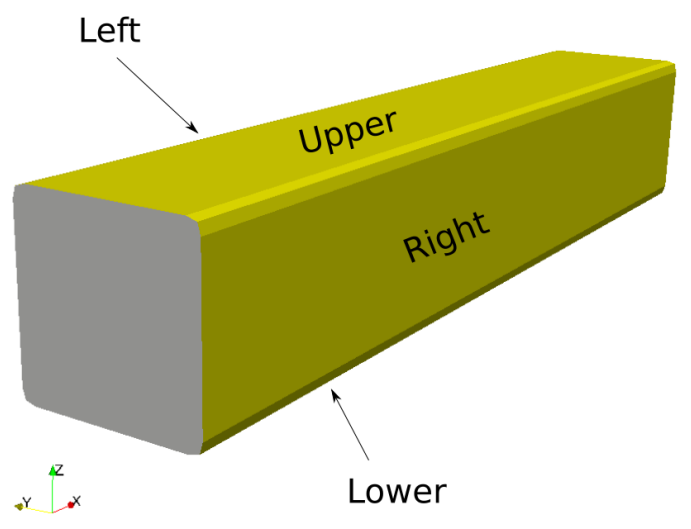

Figure 2. Illustration of the boundary patches on the fluid region

\subsection{Boundary conditions}

In the effort of analyzing what is the effect of natural convection and radiation inside the hollow beam during a fire, an artificial fire boundary condition is used outside the beam. In equation 9 the boundary condition ISO-834 curve for the warm wall is illustrated.

$$
\theta_{g}=293+345 \log \left(8\left(\frac{t}{60}\right)+1\right)
$$

in which $\mathrm{t}$ is time given in seconds and the illustration of the curve can be seen figure 3 .

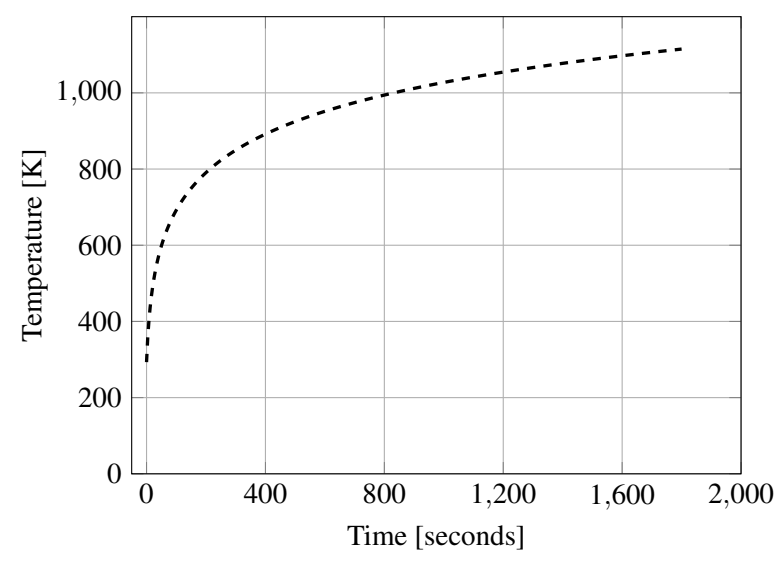

Figure 3. Illustration of the standard fire - - - in equation 9

In this paper the validity of this stand fire boundary conditions or its representation on the real fire is not studied more thoroughly. This is why an artificial temperature difference boundary condition between the warm side and cold side of the beam is used. For this study the cold side will be kept $50 \mathrm{~K}$ lower than the warm side.

Table 2. Boundary conditions used for the computational model

\begin{tabular}{|c|c|}
\hline Boundary name & Boundary condition \\
\hline \multicolumn{2}{|c|}{ Solid region } \\
\hline Warm wall & $u_{i}=0, T=$ standardfire \\
\hline Cold wall & $u_{i}=0, T=$ standardfire $-50 \mathrm{~K}$ \\
\hline Insulated ends & $\frac{\partial u_{i}}{\partial x_{n}}=\frac{\partial T}{\partial x}=0$ \\
\hline Solid-to-Fluid interface & $T_{s}=T_{f},-\lambda_{s} \frac{\partial T_{s}}{\partial n}=-\lambda \frac{\partial T_{f}}{\partial n}$ \\
\hline \multicolumn{2}{|c|}{ Fluid region } \\
\hline Insulated ends & $\frac{\partial u_{i}}{\partial x}=\frac{\partial T}{\partial x}=0$ \\
\hline Fluid-to-Solid interface & $T_{s}=T_{f},-\lambda_{s} \frac{\partial T_{s}}{\partial n}=-\lambda \frac{\partial T_{f}}{\partial n}$ \\
\hline
\end{tabular}

The temperatures are measured in specific locations on the air side close to the solid and they are illustrated in figure 4. 


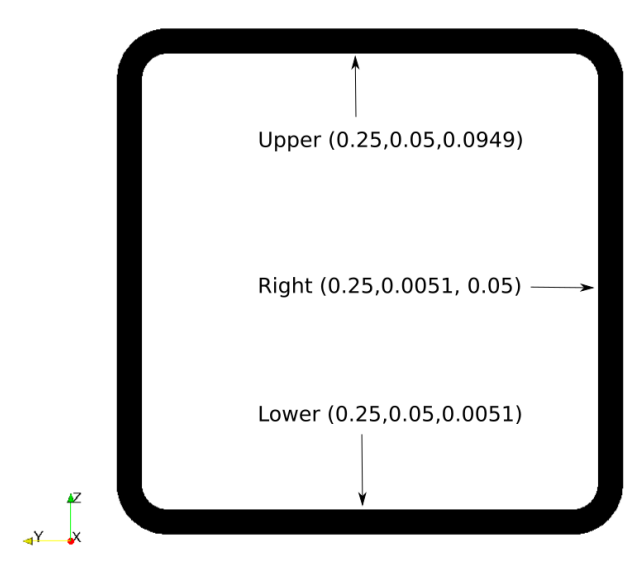

Figure 4. Illustration of the probe locations in meters

The thermal conductivity for the beam is calculated according to equation 10.

$$
\lambda_{s}=54-0.00333 * T
$$

And the specific heat for the beam is calculated according to the equation 11 .

$$
C_{p}^{s}=425+0.773 * T-0.00169 T^{2}+0.00000222 * T^{3}
$$

For the air side the specific heat capacity used was $C_{p}^{f}=1000 \frac{\mathrm{J}}{\mathrm{kgK}}$, Prandtl number 0.7 and for viscosity the sutherland approximation formula is used with the reference temperature being $110.4 K$.

\subsection{Mesh creation and independence study}

Both the air and the solid mesh were created ny using open source meshing software called Swiftblock (Nogenmyr, 2016) meshing tool. It creates structured meshes by using Blender. Blender is a 3D modelling software that is created and developed by the Blender Online Community (Community, 2017). When the blocking strategy of the mesh is created in Blender, swiftblock transforms that into a blockMeshDict which is used by a primitive meshing software called blockMesh in OpenFOAM package to finally create the computational mesh used by OpenFOAM. (Foundation, 2017) Illustration of the meshing strategy and the boundary layers at the wall in figure 5

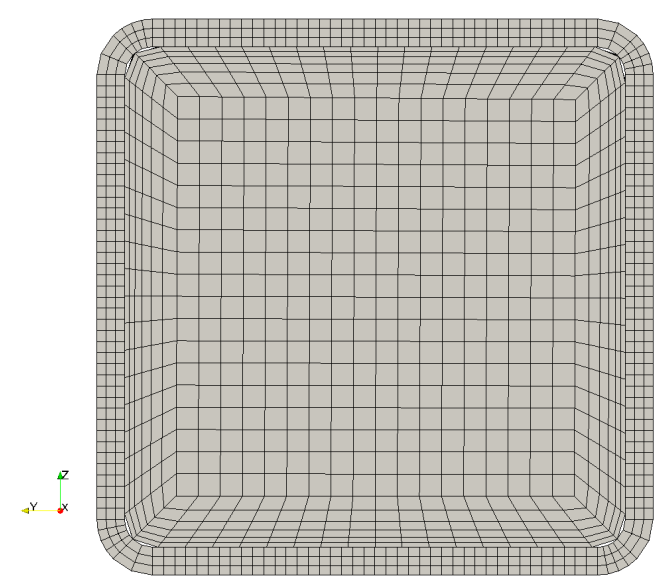

Figure 5. Illustration of the mesh structure in both fluid and solid regions

To validate that the computed results are independent on the mesh size a grid independence study was done for the mesh inside the hollow beam. Four different mesh sizes where studied and with all of them the ratio between the biggest cell size and the $y$-value at the wall was kept 3. The results can be seen in figure 6 where the convective heat transfer from the lower side of the beam is plotted against the time.

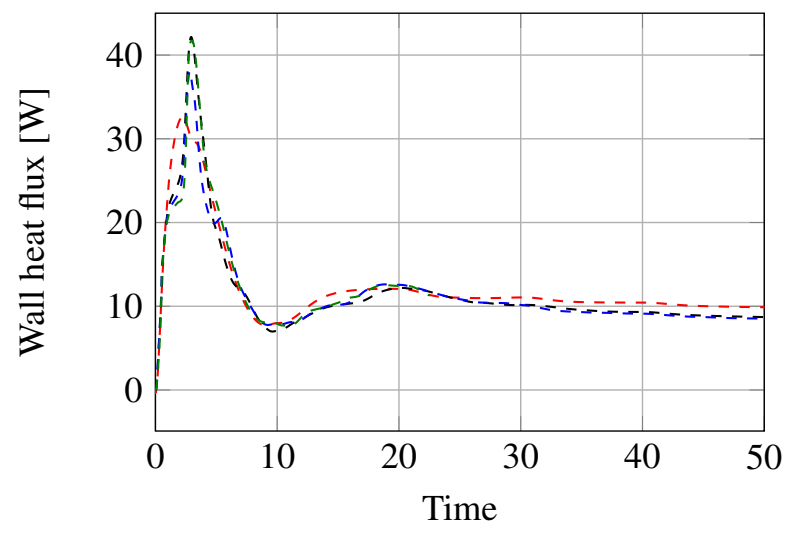

Figure 6. Convective heat flux at the lower side wall with: $5 \mathrm{k}$ ,$--- 27 \mathrm{k}---, 64 \mathrm{k}---$ and $192 \mathrm{k}-$ - - meshes $^{-}$

From the results the conclusion was made that 64000 cells was sufficient amount of cells to capture the turbulent scales of natural convection inside the beam. No turbulence models was used in the simulations.

\section{Results and Discussion}

In this section the wall heat flux through the boundary fluid to solid patch is calculated and compared between the case where the momentum equation is calculated and the case where the velocity field is frozen and not calculated through the calculation. Two differen wall heat fluxes are calculated, the other one being the flux cause by conduction and convection and is calculated according to 
the equation 5 and the other one being the radiation heat flux coming from other surfaces and is calculated according to the equation 7. In figure 7 these wall heat fluxes through the lower side of the air domain is shown.

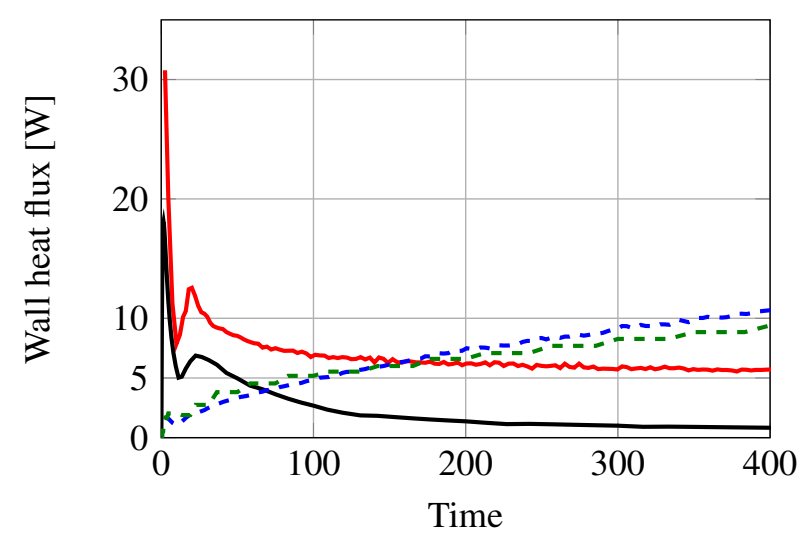

Figure 7. Wall heat flux through the lower side of the beam; conduction and convection - , conduction with frozen flow - , radiation - - - and radiation in the frozen flow - - -

The convection can be seen to dominate in the first 50 seconds of the computation but radiation will be increasing as the temperature level rise. The convection and conduction can be seen to converge after the 200 seconds, convection and conduction — being 5.5 watts and only the conduction in the frozen flow simulation — being around 0.55 watts.

The right side of the beam and the heat fluxes through that patch can be seen in figure 8 .

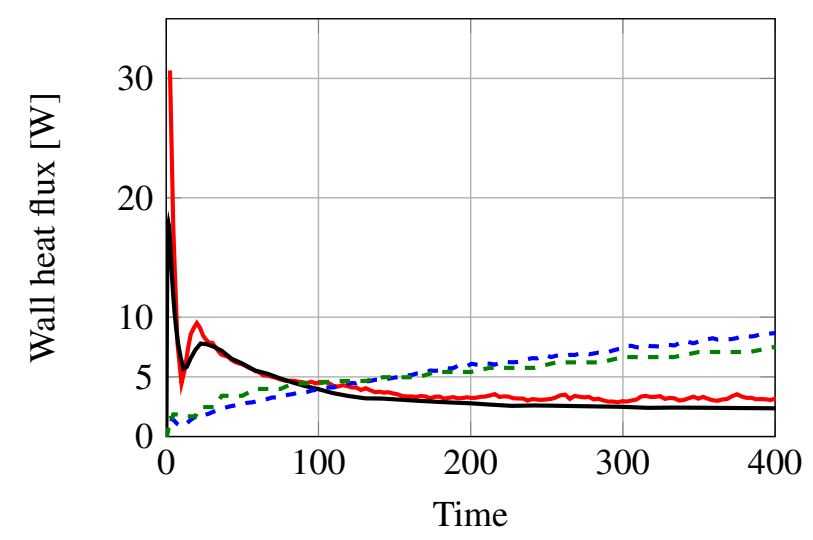

Figure 8. Wall heat flux through the right side of the beam; conduction and convection-, conduction with frozen flow - , radiation $\longrightarrow$ and radiation in the frozen flow - - -

The combined convection and conduction — and only conduction in the frozen flow simulation — can be seen to be almost equal during the first 400 seconds. The value for conduction in the frozen flow simulation — being 2.22 watts. The radiation heat flux in both of these cases can be seen to increase as the temperature increases and the absolut value being slightly higher for the case with the convection included in the simulation.
The different heat fluxes through the upper side of the beam are of course negative because the heat is going out from the air domain and the values can be seen in figure 9 .

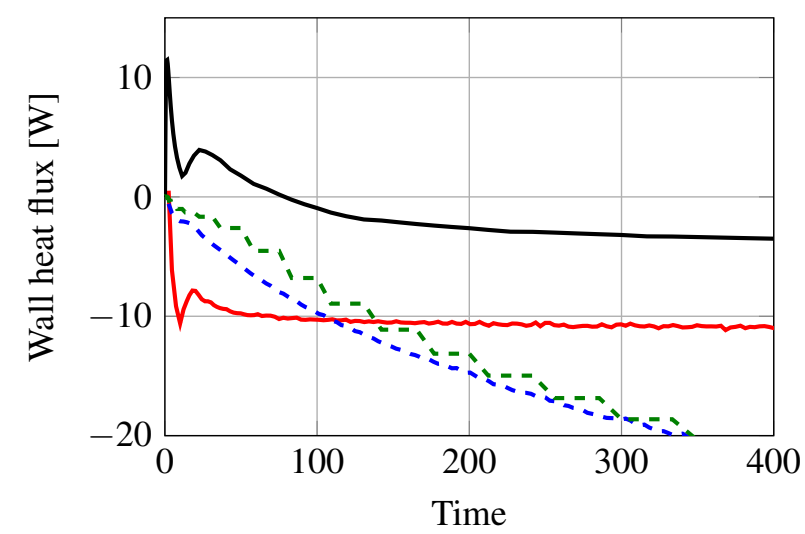

Figure 9. Wall heat flux through the right side of the beam; conduction and convection - , conduction with frozen flow - , conduction with frozen flow - , radiation - - - and radiation in the frozen flow - - -

Through the upper side of the cavity, inside the beam, the convection and conduction can be seen to converge after the first 200 seconds, convection and conduction being -10.8 watts and only the conduction in the frozen flow simulation — being around -4.2 watts. The radiation in the convection case - - - and the radiation in the frozen flow case - - - are increasing as the temperature is increasing. The sign of course being opposite because of the opposite direction of the heat.

To show what is the difference in the temperature between the case with convection, and the one without, the results can be seen in figure 10 .

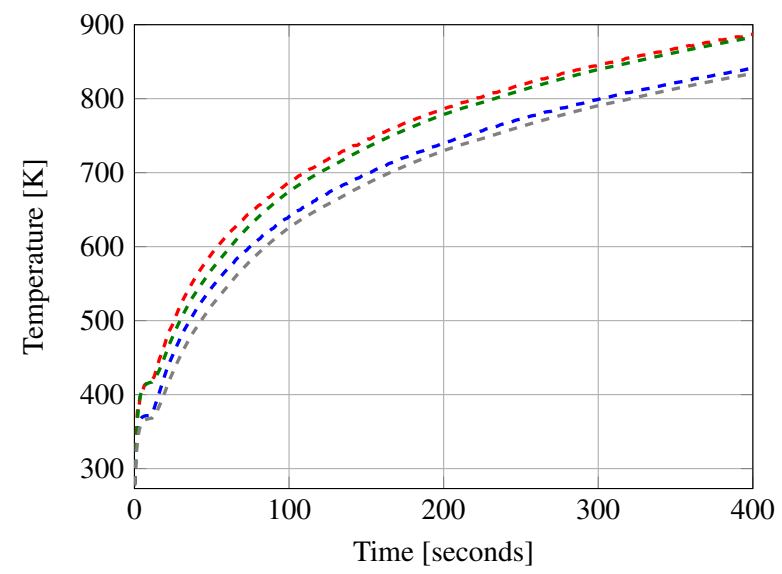

Figure 10. Temperatures in the air in the probe locations according to the figure 4: Lower side - - - , lower side frozen flow - - - , upper side - - - and upper side frozen flow - - -

The temperatures are pretty close to each other but the ones in the with convection are slighty higher than the ones wihtout. 
To illustrate that the temperature field and the wall heat flux fields inside the beam are not constant during the fire the figure 12, figure 11 and figure 13 are presented.

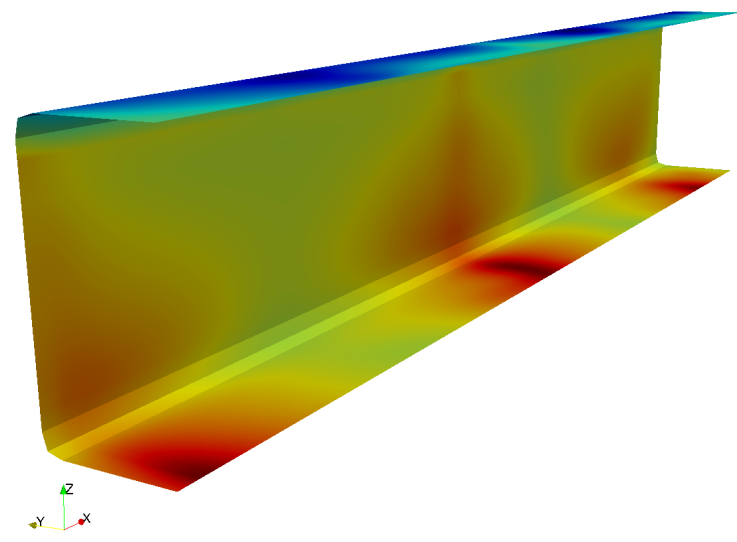

Figure 11. Illustration of the non-constant wall heat flux inside the beam when $t=400 \mathrm{~s}$

As can be seen from the figures, inside the beam a wandering recirculation zone is travelling as the simulations continues. The size of this travelling vortex is around the size of the hydraulic diameter of the insides of the hollow beam.

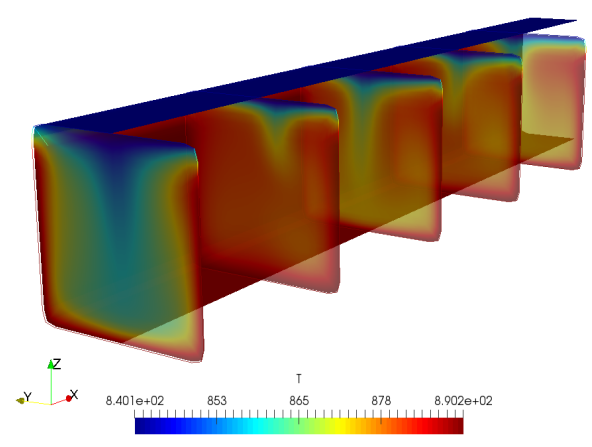

Figure 12. Illustration of the non-constant temperature field inside the beam when $t=400 \mathrm{~s}$

Final illustration of the non-constant temperature field we look at the velocity field in the middle of the beam where $x=0.25 \mathrm{~m}$ in figure 13 .

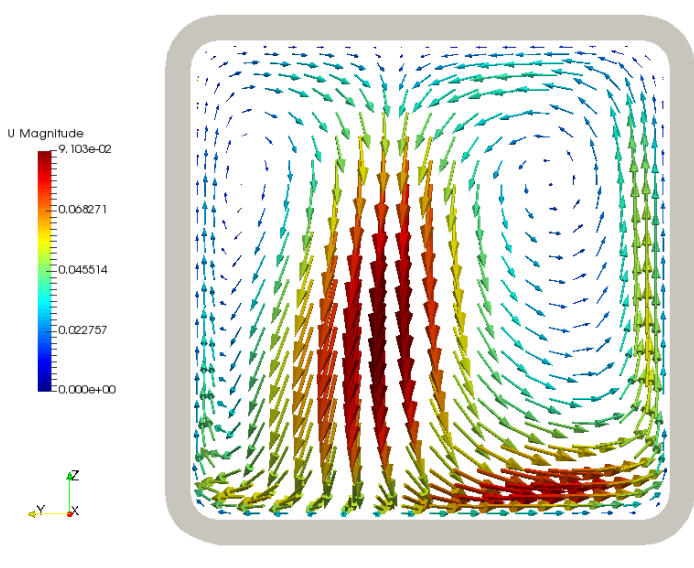

Figure 13. Illustration of the velocity field inside the beam when $t=400 \mathrm{~s}$ and $x=0.25 \mathrm{~m}$

\section{Conclusions}

In this study the effect of natural convection inside the hollow beam in a standard fire was studied. A conjugate heat transfer solver in OpenFOAM was used and all the meshes were created with open source software. To simulate what is the effect of solving the momentum and pressure equation inside the beam the case was solved with the equation and without as a frozen flow field simulation. The key findings were as follows.

1. In the beginning the convection and conduction are dominating the heat transfer.

2. After 100 seconds the radiation heat transfer is equal to the conduction and convection.

3. On the lower side of the beam the convection heat transfer is 5 times higher than conduction in the similar case with a frozen flow field simulation.

4. After 400 seconds of simulation, the radiation heat transfer is double to the amount of convection and will keep increasing as the temperature increases with the propagating fire.

As depicted in figures 11 and 12 , the internal heat transfer due to non-constant ambient temperature field causes fluid temperature variations that are meaningful when compared to ambient temperature differences. In future studies, this phenomenon will be investigated in the context of practical steel joints.

\section{References}

Blender Online Community. Blender âĂŞ a 3d modelling and rendering package, 2017. URL http://www.blender. org.

The OpenFOAM Foundation. The openfoam documentation, 2017. URL https: / / openfoam.org/. 
Tat Ching Fung, KH Tan, and Minh Phuong Nguyen. Structural behavior of chs t-joints subjected to static in-plane bending in fire conditions. Journal of Structural Engineering, 142(3): 04015155, 2015.

Shu-Bin He, Yong-Bo Shao, Hong-Yan Zhang, Dong-Ping Yang, and Feng-Le Long. Experimental study on circular hollow section (chs) tubular k-joints at elevated temperature. Engineering Failure Analysis, 34:204-216, 2013.

Markku Heinisuo and Timo Jokinen. Tubular composite columns in a non-symmetrical fire. Inzenerno-Stroitel'nyj Zurnal, (5):107, 2014.

William Morrow Kays. Convective heat and mass transfer. Tata McGraw-Hill Education, 2012.

Anthony F Mills. Basic heat and mass transfer. Pearson College Div, 1999.

Folkersma M Nogenmyr, K.J., 2016. URL https: //openfoamwiki.net/index.php/Contrib/ SwiftBlock.

E Ozyurt, YC Wang, and KH Tan. Elevated temperature resistance of welded tubular joints under axial load in the brace member. Engineering Structures, 59:574-586, 2014.

Yongbo Shao, Yijie Zheng, Haicheng Zhao, and Dongping Yang. Performance of tubular t-joints at elevated temperature by considering effect of chord compressive stress. ThinWalled Structures, 98:533-546, 2016.

MB Wong. Temperature analysis of partially heated steel members in fire. Journal of Constructional Steel Research, 128: 1-6, 2017.

Hua Yang, Faqi Liu, and Leroy Gardner. Performance of concrete-filled rhs columns exposed to fire on 3 sides. Engineering Structures, 56:1986-2004, 2013.

J Yang, YB Shao, and C Chen. Experimental study on fire resistance of square hollow section (shs) tubular t-joint under axial compression. Advanced Steel Construction, 10(1):7284, 2014. 